\title{
Feasibility and utility of transcutaneous spinal cord stimulation combined with walking-based therapy for people with motor incomplete spinal cord injury
}

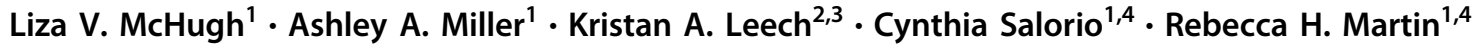

Received: 12 June 2020 / Revised: 21 October 2020 / Accepted: 22 October 2020

(c) The Author(s) 2020. This article is published with open access

\begin{abstract}
Study design Prospective case series.

Objectives To evaluate the feasibility and preliminary efficacy of combining transcutaneous spinal cord stimulation (TSCS) with walking-based physical therapy.

Setting Hospital-based outpatient center in Maryland, United States.

Methods Ten individuals with chronic ( $>1$ year) motor incomplete spinal cord injury (iSCI) completed 23 sessions of 2-h therapy over 8 weeks. TSCS was delivered for the first $30 \mathrm{~min}$ of each session using a clinically available device with adjustable current. To assess feasibility of the intervention, we tracked pain, adverse events, and participant retention. Preliminary efficacy was assessed by evaluating changes in walking speed, endurance, and quality following the intervention with select functional outcome measures (10-m walk test (10MWT), 6-min walk test (6MWT), timed up and go, and walking index for spinal cord injury II).

Results We found that the combined intervention was feasible in an outpatient clinical setting. Participants tolerated the TSCS well, with no reports of significant adverse events or other issues (e.g., skin irritation or pain that disrupted training). None of the participants elected to discontinue the study. Participants also showed significant improvements in each measure of walking function following the intervention. Changes in walking speed, as measured by the 10MWT $(0.56 \pm 0.29 \mathrm{~m} / \mathrm{s}$ to $0.72 \pm 0.36 \mathrm{~m} / \mathrm{s}$ ), exceeded the minimal clinically important difference for individuals with iSCI. Changes in walking quality and endurance, as measured by the 6MWT $(149.88 \pm 99.87 \mathrm{~m}$ to $194.53 \pm 106.56 \mathrm{~m})$, exceeded the minimal detectable change for individuals with iSCI.

Conclusions These results indicate that TSCS is clinically feasible and may be useful as an adjunct to walking-based therapy for adults with iSCI.
\end{abstract}

Rebecca H. Martin

martinre@kennedykrieger.org

1 International Center for Spinal Cord Injury, Hugo W. Moser Research Institute at Kennedy Krieger Institute, Baltimore, MD 21205, USA

2 Department of Neuroscience, The Johns Hopkins University School of Medicine, Baltimore, MD 21205, USA

3 Division of Biokinesiology and Physical Therapy, University of Southern California, Los Angeles, CA 90007, USA

4 Department of Physical Medicine and Rehabilitation, The Johns Hopkins University School of Medicine, Baltimore, MD 21205, USA

\section{Introduction}

Spinal cord injury (SCI) disrupts the transmission of motor and sensory information through the spinal cord. The majority of injuries are motor incomplete spinal cord injury (iSCI) and result in walking dysfunction [1]. Recovery of walking function is a high priority among individuals iSCI and is a common target of physical therapy [2]. Despite significant advances in the field, recovery of independent walking remains elusive for most patients with iSCI.

Intensive gait training is considered the most effective intervention to improve walking function following iSCI $[3,4]$. Studies show that this training results in clinically meaningful improvements in gait speed, endurance, balance, and lower extremity strength $[1,5,6]$. However, even 
with these improvements, significant deficits in walking function persist.

In an effort to potentiate gains in walking function than can be achieved with gait training, previous work in animal and human models has explored pairing intensive gait training with neuromodulatory interventions [7]. One adjuvant intervention that has gained recent attention is spinal cord electrical stimulation. The most well studied form of spinal electrical stimulation to promote recovery of walking function is surgically implanted lumbosacral epidural stimulation [7-10], which acts by directly stimulating the dorsal nerve roots to increase the excitability of interneuronal networks involved in the control of locomotion [7, 10-14]. The current literature has largely described epidural stimulation in individuals with complete SCI, demonstrating that lumbosacral epidural stimulation alone can facilitate reciprocal, step-like movements, and when used in combination with intensive locomotor training can lead to improved walking abilities [8, 14-17].

While this work is exciting, there are limitations to the feasibility of epidural stimulation. First, the surgical placement of the stimulator is invasive and inherently risky $[10,14,15]$. Second, studies demonstrating efficacy of epidural stimulation are time intensive, describing extensive locomotor and gait training for 20-85 weeks after stimulator implantation [8, 10, 14]. Finally, while all participants showed improvement in voluntary motor control, not all recovered durable walking function $[10,14,15]$.

Fortunately, a noninvasive form of spinal electrical stimulation has also been shown to impact spinal excitability in individuals with SCI [18-20]. Transcutaneous spinal cord stimulation (TSCS) is thought to increase the excitability of spinal locomotor circuits through dorsal root afferents $[18,21,22]$. Research suggests that this change in excitability enables the brain to utilize functionally silent descending pathways to produce and enhance voluntary movements of paretic limbs [18-20]. Application of TSCS, both in single sessions and repeated over at least 4 weeks, improves standing postural control, gait kinematics, and spinal motor output, when paired with treadmill gait training [17-20]. This suggests that TSCS may be an effective, noninvasive intervention to augment the effects of gait training in motor iSCI.

Despite literature supporting the benefits of TSCS, the effects of pairing TSCS with walking-based therapy in a clinical setting are rarely investigated and largely unknown. Stimulators used in some previous studies utilize a proprietary waveform, not clinically available. The biphasic rectangular wave of these stimulators includes a carrier frequency of $10 \mathrm{kHz}$, reported to make the stimulation more comfortable than traditional biphasic waveforms [19, 21]. Clinically available stimulators may elicit the same effects, but to date these have not been studied. The purpose of this study was to assess the feasibility and collect preliminary data on the efficacy of providing TSCS with a clinically available waveform as a combined approach to intensive walking-based therapy in an outpatient setting in individuals with motor iSCI.

\section{Methods}

\section{Participants}

Participants were recruited from the International Center for Spinal Cord Injury at Kennedy Krieger Institute. Inclusion criteria for the study were: (1) 18-65 years old; (2) >1 year post SCI; (3) nonprogressive SCI; (4) neurological level at or above $\mathrm{T} 10$; (5) tolerates upright position for $>30 \mathrm{~min}$; (6) medically stable (no hospitalizations in last 3 months); (7) able to comply with procedures and follow-up; and (8) are legally able to make their own healthcare decisions. Exclusion criteria for participation in the study were as follows: (1) open wounds at stimulation site; (2) pregnant women; (3) ROM limitations impacting gait training; (4) cardiac pacemaker/defibrillator; (5) active cancer diagnosis; (6) currently receiving TSCS; (7) evidence of uncontrolled autonomic dysreflexia; or (8) non-English speaking. Participants were asked to not make any changes to their medications for the duration of the study.

\section{Study design}

This prospective case series was a within-participant, repeated measures design (Fig. 1). The intervention

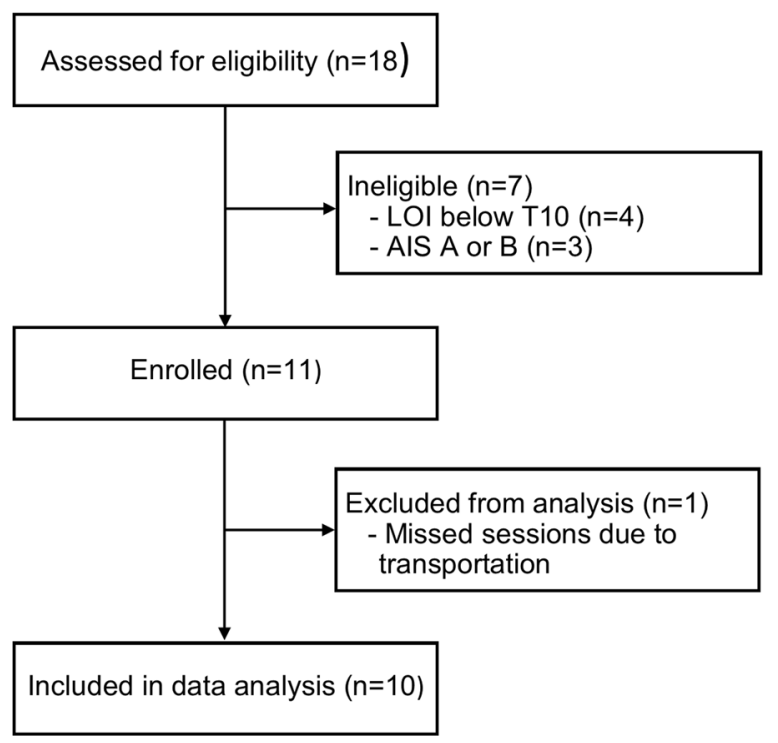

Fig. 1 Timeline of training sessions and assessments of walking function. 
consisted of a total of 23 training sessions. Measures of feasibility, pain, skin response, treatment time, and adverse events were collected every session. Measures of walking function were completed at baseline and every sixth session. Post-testing occurred within $48 \mathrm{~h}$ of the final training session. All testing was completed without stimulation.

\section{Walking-based therapy}

Therapy sessions were provided for 2 hours three times per week for 8 weeks. TSCS was provided for the first 30 minutes, concurrent with gait activities. Gait activities continued for the remaining 90 minutes without stimulation. This session duration matches other walking interventions with established efficacy, while decreasing the frequency slightly to reduce the burden to the participant, optimize participant retention, and most closely approximate a typical outpatient plan of care [1,23].

Training sessions were implemented by one of five licensed physical therapists. All therapists followed a general walking-based training protocol with flexibility to address participant-specific gait impairments. The primary focus of each session was walking function.

Intervention activities were divided into four categories: gait-based activities, functional task practice, strengthening exercises, and "other". Gait-based activities included treadmill walking with body weight support (BWS), overground walking with and without BWS, and higher level dynamic gait activities, like tandem walking, step-ups, and marching, walking around obstacles and over uneven surfaces. Functional task practice included the performance of functionally relevant tasks, such as sit to stands from a chair or couch, target stepping, outdoor balance activities, and stair negotiation. Strengthening exercises consisted of a variety of resistance exercises targeting the core and lower extremity musculature. Lastly, "other" accounted for time spent testing, set-up, and participant initiated rest breaks.

\section{Transcutaneous spinal cord stimulation}

At the start of each session, TSCS was applied using a $5 \times$ $10 \mathrm{~cm}$ oval electrode placed midline on the skin between spinous processes $\mathrm{T} 11$ and $\mathrm{T} 12$ and two $7.5 \times 13 \mathrm{~cm}$ rectangular electrodes placed symmetrically on the skin over the lower abdomen [18, 21]. The Vectra Neo (Chattanooga; Hixson, $\mathrm{TN}$ ) was used to deliver a symmetrical biphasic rectangular waveform, at $50 \mathrm{~Hz}$ and $1 \mathrm{~ms}$, for 30 continuous minutes of stimulation [18]. In previously published work, treatment frequencies of $20-50 \mathrm{~Hz}$ were associated with alternating flexion/extension patterns in EMG [16]. In pilot trials, we stimulated at 20,30 , and $50 \mathrm{~Hz}$. At $50 \mathrm{~Hz}$, participants could tolerate greater intensities as compared with 20 or $30 \mathrm{~Hz}$. Though current literature indicates $50 \mathrm{~Hz}$ may preferentially target spasticity [24], we did not observe a difference in performance at each frequency. We elected to use $50 \mathrm{~Hz}$ to minimize potential discomfort. Intensity was set at the beginning of each session to individual tolerance or submotor threshold, whichever was less. Intensities ranged from 20 to $80 \mathrm{~mA}$ and were consistent within sessions but varied between sessions, depending on the participant and their tolerance. Stimulation was adjusted with the patient in sitting, typically at the edge of the mat. Intensity was adjusted to participant's tolerance or until they reported tingling in the lower extremities. In participants with unreliable sensation, stimulation intensity was increased until oscillating lower extremity movement was observed. Intensity was decreased slightly if the oscillations disrupted the participant's voluntary motion or efficient gait pattern. Once stimulation was optimized, participants engaged in training as outlined above. Training continued following the stimulation period for the 90-min remainder of the session to take advantage of the priming and lasting neuromodulatory effects provided by the TSCS [19, 21, 24].

\section{Outcome measures}

\section{Assessment of feasibility and safety}

Pain scores were measured before and after the session using the Numeric Rating Scale for Pain (NRS for Pain) [25]. Pain was rated on a scale of $0-10$, with 0 being no pain at all and 10 being the worst pain imaginable [25]. Significant adverse events including, but not limited to, fall incidence, episodes of autonomic dysreflexia, and cardiovascular events were recorded for the duration of the study. Skin response to TSCS was documented at the end of each session to assess skin tolerability. The skin was inspected for signs of mottling or non-blanchable erythema under and around the electrode site to rule out possible tissue injury.

The percentage of possible sessions attended was used as a measure of compliance with the intervention. Therapists' opinions on ease of administration and comparison to traditional gait-based therapy were also collected.

\section{Assessment of effects on walking function}

Changes in walking function were assessed with the 10-m walk test (10MWT), 6-min walk test (6MWT), timed up and go (TUG), and walking index for spinal cord injury II (WISCI-II). The 10MWT, 6MWT, and TUG are commonly and widely used functional ambulation outcome measures and have good test-retest, interobserver reliability, and construct validity in ambulatory SCI [26-30]. The WISCIII, developed specifically for the SCI population, is used to describe walking impairment [27] and provides a more comprehensive consideration of the use of braces and 
assistive devices not found in the other measures. These measures were chosen to be consistent with a 2008 recommendation of the National Institute on Disability and Rehabilitation Research, to best demonstrate gait improvements in participants with SCI, and the Common Data Elements recommended by the National Institute of Neurological Disease and Stroke and the National Institute of Health [31-33]. For each test, participants were allowed to use a preferred assistive device and/or lower extremity orthoses as needed. Each participant used the same device or bracing at all assessment sessions. All testing was completed without TSCS and participants were not provided any physical assistance during testing. Outcome measures were performed in the same order at each testing point across participants. Participants were offered rest breaks between outcomes as needed.

\section{Data analysis}

Measures of feasibility are detailed at a group (presented as means \pm standard deviations) or individual level, as appropriate. To evaluate changes in walking function following the intervention, we performed separate paired comparisons of pre- and post-intervention assessments for each outcome measure of interest. Paired $t$-tests were used for continuous variables (i.e., 10MWT, 6MWT, and TUG) and Wilcoxon signed-rank was used for ordinal variables (i.e., WISCI-II). For all analyses, $\alpha$ level was set to 0.05 . Finally, to characterize the time course of changes in walking function, we also present the change scores for each measure at specific time points (visits 6,12 , and 18) during the intervention. These changes are described relative to the established minimal detectable change (MDC) or minimal clinically important difference (MCID) for each measure.

\section{Results}

Eighteen people were screened and 11 were enrolled (Fig. 2). We set an a priori threshold to exclude participants from analysis if they missed $10 \%$ of the training sessions; one subject was eliminated (missed sessions due to transportation), leaving a final sample of ten. The sample was heterogeneous with respect to neurological level, AIS classification, age, time since injury, and gender. Demographic information is shown in Table 1.

\section{Feasibility data}

Reported pain levels ranged from 0 to 4 on the NRS for Pain with an average of $0.12 \pm 0.27$ across all participants and sessions, suggesting participants experienced minimal to no pain during the TSCS and associated gait activities. There were no instances in which reports of pain (anything $>0$ ) necessitated termination of the stimulation duration or modification of the interventions.

No significant adverse events, including but not limited to falls, injury, autonomic dysreflexia, or related illness, were reported or observed over the course of the study. Mild, blanchable, erythema under the electrodes was noted on occasion, as is consistent with surface electrical stimulation. These mild skin responses did not limit stimulation duration or impact the physical intervention. No durable or
Fig. 2 Participant recruitment diagram.

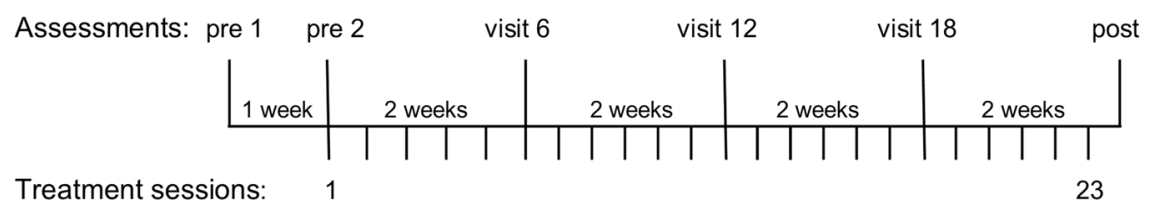

Table 1 Demographics.

\begin{tabular}{lllllll}
\hline Subject & Gender & $\begin{array}{l}\text { Age } \\
\text { (years) }\end{array}$ & $\begin{array}{l}\text { Time since injury } \\
\text { (years) }\end{array}$ & $\begin{array}{l}\text { Cause } \\
\text { of injury }\end{array}$ & Neurological LOI & AIS classification \\
\hline 01 & M & 64 & 3 & Non & T3 & D \\
02 & F & 22 & 2 & Non & T8 & C \\
03 & M & 52 & 11 & Trauma & C6 & D \\
04 & M & 63 & 57 & Trauma & T1 & D \\
05 & M & 55 & 18 & Non & T4 & D \\
06 & F & 28 & 2 & Trauma & C4 & D \\
07 & F & 22 & 6 & Non & C5 & C \\
08 & M & 40 & 20 & Trauma & C5 & D \\
09 & F & 60 & 12 & Non & T9 & C \\
10 & M & 24 & 3 & Non & C7 & C \\
\hline
\end{tabular}




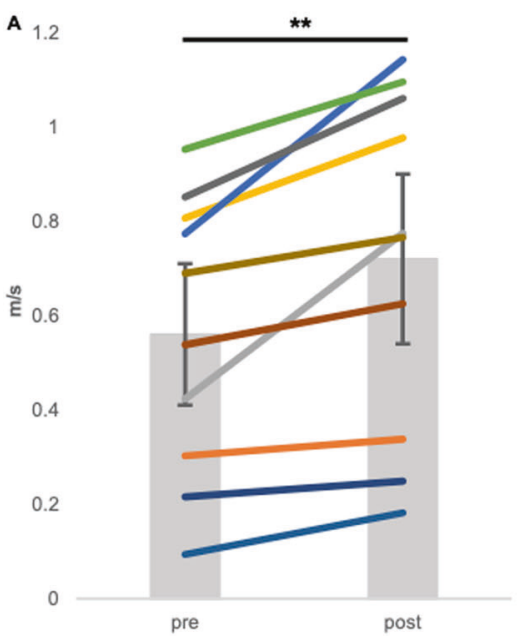

Fig. 3 Measures of walking function pre- and post-intervention. Following the interventions, participants demonstrated improvements in walking function demonstrated by significant changes in 10-m walk
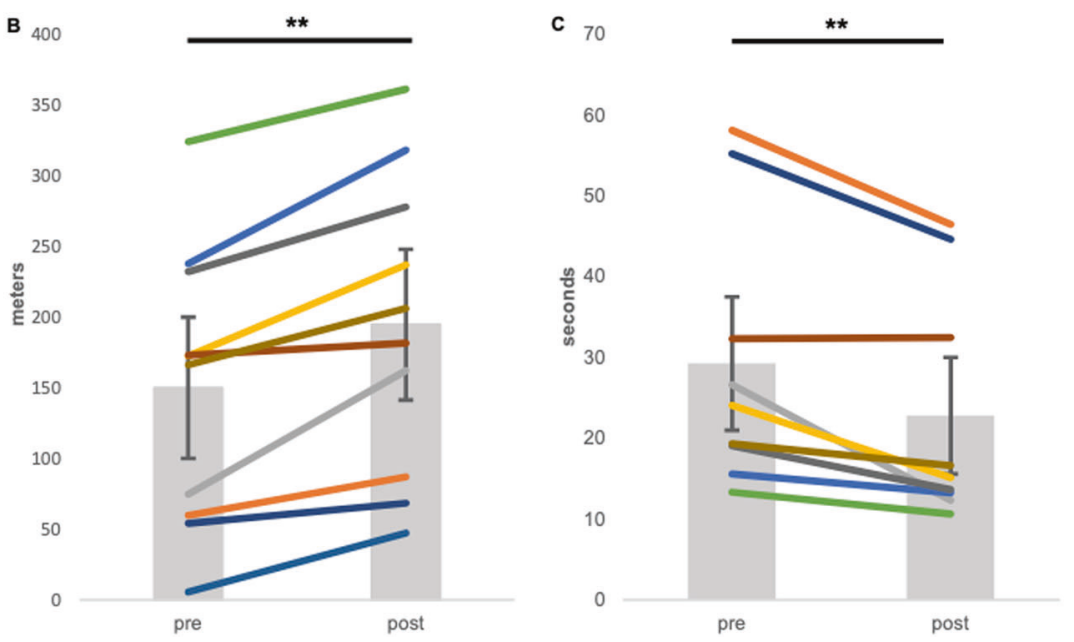

test $(\mathbf{A} ; n=10), 6$-min walk test $(\mathbf{B} ; n=10)$, and timed up and go $(\mathbf{C}$; $n=9$ ). Data are presented as mean \pm SD. **Indicates $p<0.0001$.

Lines on each plot represent individual data.
Fig. 4 Changes in WISCI-II pre- and post-intervention. Participants demonstrated significant improvements in the walking index for spinal cord injury II $(* p=0.014)$. Group mean changes $(\mathbf{A}, n=10)$ and individual changes $(\mathbf{B}, n=10)$ across the 8 weeks of training are depicted.

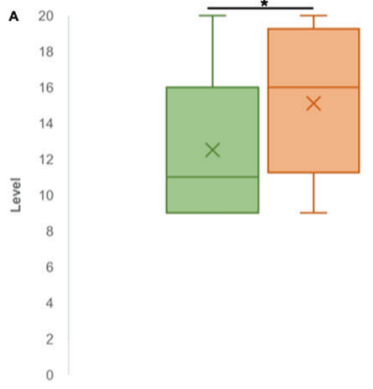

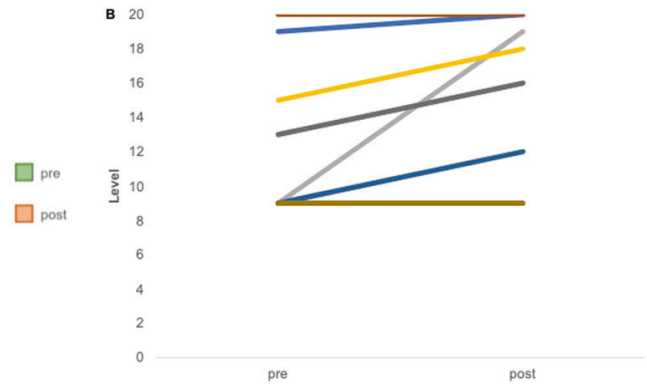

mottled erythema, blisters, or other skin irritation was noted in any participant.

Ten participants completed the 8-week intervention and associated testing. No participant elected to discontinue the study. Participants completed $97.5 \%$ of sessions. Only sessions $>90$ min were considered complete for the purposes of analysis. Average duration of session across all participants was $112.3 \pm 18.7 \mathrm{~min}$ of an anticipated $120 \mathrm{~min}$ session. Three participants (06, 10, and 11) missed one session for cold symptoms, prearranged vacation, and transportation difficulties. Three participants $(02,07$, and 08) stopped sessions early, after $90 \mathrm{~min}$, due to episodes of incontinence. All episodes of incontinence were $60 \mathrm{~min}$ or more after completion of the stimulation. Incontinence never occurred during stimulation nor impacted willingness to participate.

Therapists reported that the set-up and administration of the stimulation was easy. They were all familiar with the stimulator from other clinical uses. Therapists reported that the stimulation did not limit or hinder any gait interventions. An additional staff person was required to move the stimulation unit (on wheels) when participants required physical assist for intervention activities.

\section{Preliminary effectiveness data}

Figures 3 and 4 show pre- and post-intervention measures of walking function. Participants exhibited significant improvements in all measures of walking function following the intervention. Specifically, we observed a significant increase in gait speed as measured by the 10MWT, $0.56 \pm$ $0.29 \mathrm{~m} / \mathrm{s}$ to $0.72 \pm .36 \mathrm{~m} / \mathrm{s},(t(9)=-4.08, p<0.0001$; Fig. $3 \mathrm{~A}$ ), and endurance as measured by the 6MWT, 149.88 \pm $99.87 \mathrm{~m}$ to $194.53 \pm 106.56 \mathrm{~m},(t(9)=-5.42, p<0.0001$; Fig. 3B). Improvements in functional mobility (i.e., transitional movements, balance, gait speed, etc.) were captured by significant changes in the TUG $(t(8)=3.88, p<0.0005$; Fig. 3C). One participant (07) was excluded from the TUG analysis, as she was unable to independently complete the required sit to stand transition at baseline, but she showed improvement from session 6 to post-intervention of $53.75 \mathrm{~s}$. Finally, we found a significant group increase on the WISCI-II $(Z(9)=-2.46, p<0.05$; Fig. 4A), suggesting reduction in physical assistance or use of a less restrictive adaptive device or bracing option (i.e., walker to crutches; braces to no braces). Seven out of ten participants improved their individual WISCI-II score by at least 1 point (Fig. 4B), 

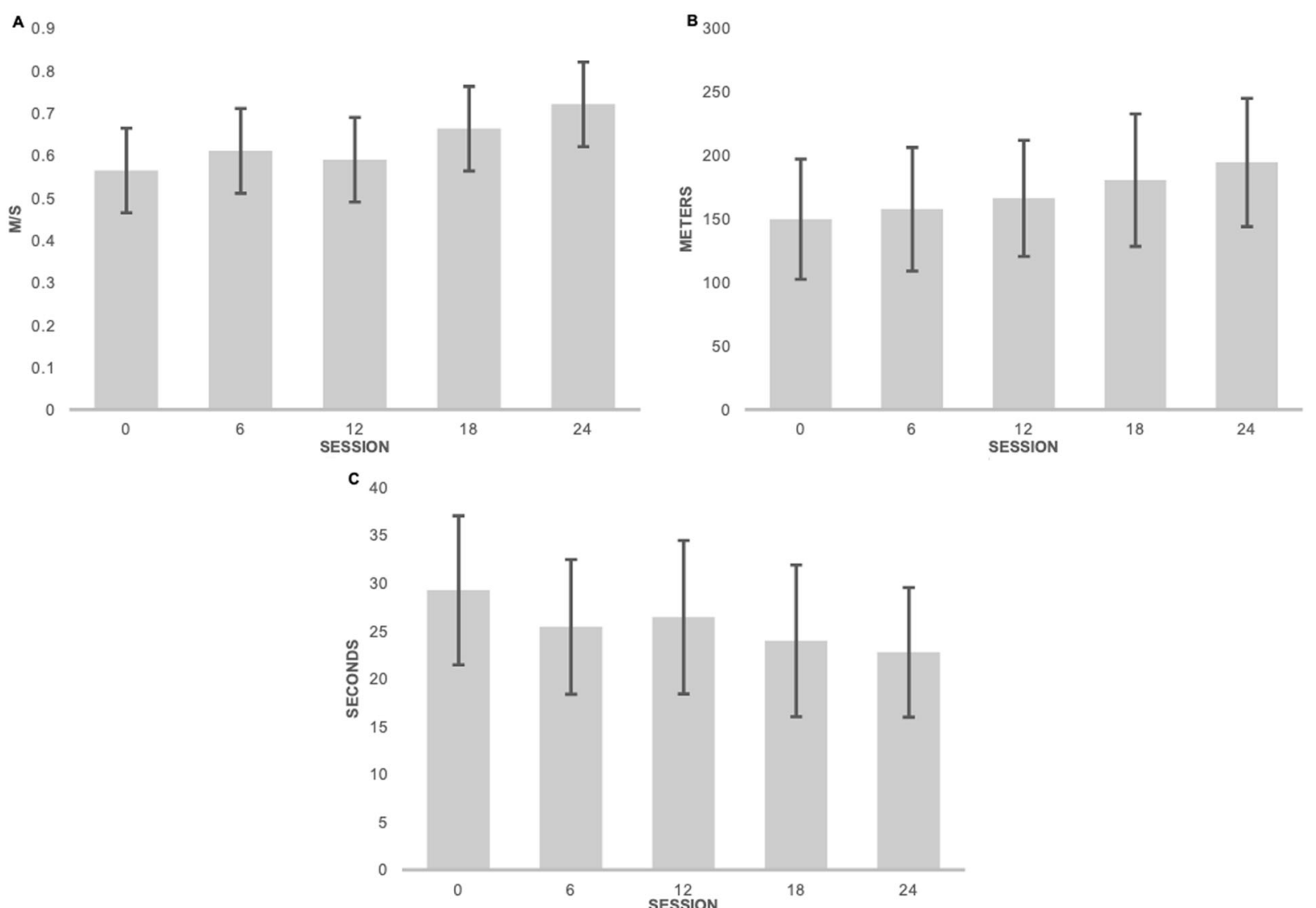

Fig. 5 Changes in walking function throughout the intervention. Panels depict the group average $($ mean \pm SD) performance in the 10MWT (A, $n=10)$, 6MWT $(\mathbf{B}, n=10)$, and TUG $(\mathbf{C}, n=9)$ at

indicating improvement in walking capacity. All participants increased or maintained their score; one participant achieved the maximum score at baseline, precluding improvement on this measure.

\section{Time course of changes}

Changes in walking functions at specific time points (sessions 6, 12, 18, and post-intervention) are displayed in Fig. 5. By session 18, group mean changes in gait speed exceeded the MCID reported for the 10MWT in individuals with iSCI $(0.06 \mathrm{~m} / \mathrm{s}$; Fig. 5A) [34]. This improvement was consistent throughout the group with eight out of ten participants' individual changes pre- and post-intervention exceeding the MCID. Meaningful changes in endurance occurred earlier in the course of the intervention, with group mean changes in the 6MWT exceeding the MDC for individuals with iSCI by session 12 (54.8 m [26, 35]; Fig. 5B). Changes in walking capacity, as measured by the WISCI-II, also occurred early in the course of the intervention. By session 6, group mean changes exceed the MDC (1 level; [36]) for people with SCI. While this change occurred early, participants continued to improve over the course of the 2-week intervals over the course of the intervention. Participants demonstrated change in the 10MWT and 6MWT that exceed the MCID for each measure at or before session 18.

intervention, with a group mean improvement of 2.6 levels on WISCI-II by the post-intervention assessment.

Changes in the TUG across time were more modest. Group mean change, although statistically significant, did not exceed the established MDC for people with SCI (10.8 s; Fig. 5C) [29], suggesting that participants' walking function improved but their ability to perform transitional movements and specific strength may not have.

\section{Discussion}

In this study, we explored the feasibility of implementing a TSCS protocol in combination with walking-based therapy in individuals with motor iSCI. We provided TSCS through a commercially available electrical stimulation device for the first 30 minutes of each 2-hour session provided three times per week for 8 weeks. Walking-based interventions continued after the stimulation to take advantage of the lasting stimulation-induced changes in spinal excitability $[18,20]$.

We found that the use of TSCS in combination with walking-based physical therapy is feasible and safe in an 
outpatient clinical setting. Participants completed 8 weeks of this combined training without report or observation of significant adverse events, despite considerable diversity in clinical characteristic of the sample. The finding that TSCS was feasible and safe in varying degrees of injury enhances its clinical application.

We investigated TSCS with a symmetrical, biphasic waveform via a clinically available device. Previous studies have used an investigational waveform and proprietary device purported to be more tolerable [18, 19, 21]. None of our participants complained of pain nor cited pain as limiting the stimulation intensity or duration, suggesting TSCS may be feasible with a safe, available waveform, enhancing its clinical utility and accessibility for therapists. Electrophysiological studies are still necessary to confirm the neuromodulatory mechanisms of different waveforms currently under investigation.

These results provide preliminary evidence for the effectiveness of this combined training approach to improve walking function in individuals with chronic iSCI. We found that pre- and post-intervention groups mean changes for the 10MWT, WISCI-II, 6MWT met or exceeded the established MDC or MCID for each measure of walking function [26, 34-36]. Many locomotor and activity-based interventional studies for subacute or chronic iSCI report improvement in walking function, specifically related to speed, with similar magnitudes, but often over much longer periods of time $[6,16,37]$. Our participants had been deemed at their maximum functional capacity in traditional therapy, but we demonstrate that that their performance increased at each testing time point, suggesting it is possible for individuals to experience additional real change and progression if training is prolonged. Further research is needed to determine the most effective stimulation waveform, paradigm, and appropriate training dosing and duration, especially in the context of limited healthcare resources.

This was a small, non-powered, non-blinded pilot study and several limitations should be considered when interpreting these data. First, although the changes we observed are significant and most exceed the MCID/MDC for individuals with chronic iSCI, without a control group we cannot attribute these changes to the combined approach versus therapy alone. It is also not clear which factors (age, American Spinal Injury Association Impairment Scale, Neurological Level, baseline walking speed, etc.) predict participants' improvement in response to the intervention. A larger sample would allow for subgroup analysis, which may elucidate predictive factors. Furthermore, participants engaged in a variety of tasks within a single session, which is typical of clinical therapy sessions. Future studies might limit the walking-based therapy interventions to determine those which are most effective. Finally, as with any intervention in this population, incontinence and transportation are issues. While not related to the intervention, these were issues in our study and could have an impact on the results. Across all sessions, there were three instances of incontinence that occurred more than $30 \mathrm{~min}$ after stimulation. Participants did not attribute the episodes of incontinence to the intervention, as incontinence is a common complication of iSCI. Still, we cannot rule this out as an adverse event and it should be more closely investigated in future studies.

These data indicate that TSCS is both clinically feasible and may be a useful adjunct to walking-based therapy for individuals with motor iSCI. Further work is required to adequately assess the benefits of using TSCS combined with physical rehabilitation to improve long-term walking function post iSCI, compared with physical therapy alone.

\section{Data availability}

The data sets generated and/or analyzed during the current study are available from the corresponding author on reasonable request.

Acknowledgements The authors wish to thank the Orokawa Family Foundation for their gift to fund this research and Scott Meyer, PTA, Marjorie Morgan, PTA, and Lauren Conroy, PT, for their assistance in data collection. The authors are also grateful for the support and guidance in study completion and manuscript preparation from Amy Bastian, PhD, PT, and Cristina Sadowsky, MD.

Funding This study was funded by a directed donation from The Orokawa Foundation and Institutional support.

\section{Compliance with ethical standards}

Conflict of interest The authors declare that they have no conflict of interest.

Ethical approval We certify that all applicable institutional and governmental regulations concerning the ethical use of human volunteers were followed during the course of this research. The study was approved by the Johns Hopkins Institutional Review Board.

Informed consent All participants provided informed consent prior to participating.

Publisher's note Springer Nature remains neutral with regard to jurisdictional claims in published maps and institutional affiliations.

Open Access This article is licensed under a Creative Commons Attribution 4.0 International License, which permits use, sharing, adaptation, distribution and reproduction in any medium or format, as long as you give appropriate credit to the original author(s) and the source, provide a link to the Creative Commons license, and indicate if changes were made. The images or other third party material in this article are included in the article's Creative Commons license, unless indicated otherwise in a credit line to the material. If material is not included in the article's Creative Commons license and your intended 
use is not permitted by statutory regulation or exceeds the permitted use, you will need to obtain permission directly from the copyright holder. To view a copy of this license, visit http://creativecommons. org/licenses/by/4.0/.

\section{References}

1. Harkema S, Behrman A, Barbeau H. Evidence-based therapy for recovery of function after spinal cord injury. In: Verhaagen $\mathrm{J}$, McDonald JW, editors.Handbook of clinical neurology. Amsterdam: Elsevier; 2012. p. 259-74.

2. Anderson KD. Targeting recovery: priorities of the spinal cordinjured population. J Neurotrauma. 2004;21:1371-83.

3. Field-Fote EC. Spinal cord control of movement: implications for locomotor rehabilitation following spinal cord injury. Phys Ther. 2000;80:477-84.

4. Jones ML, Evans N, Tefertiller C, Backus D, Sweatman M, Tansey K, et al. Activity-based therapy for recovery of walking in chronic spinal cord injury: results from a secondary analysis to determine responsiveness to therapy. Arch Phys Med Rehabil. 2014;95:2247-52.

5. Labruyère R, van Hedel HJA. Strength training versus robotassisted gait training after incomplete spinal cord injury: a randomized pilot study in patients depending on walking assistance. $\mathbf{J}$ Neuroeng Rehabil. 2014;11:4.

6. Morrison SA, Lorenz D, Eskay CP, Forrest GF, Basso DM. Longitudinal recovery and reduced costs after 120 sessions of locomotor training for motor incomplete spinal cord injury. Arch Phys Med Rehabil. 2018;99:555-62.

7. Gerasimenko Y, Roy RR, Edgerton VR. Epidural stimulation: comparison of the spinal circuits that generate and control locomotion in rats, cats and humans. Exp Neurol. 2008; 209:417-25.

8. Gill ML, Grahn PJ, Calvert JS, Linde MB, Lavrov IA, Strommen JA, et al. Neuromodulation of lumbosacral spinal networks enables independent stepping after complete paraplegia. Nat Med. 2018;24:1677-82.

9. Mayr W, Krenn M, Dimitrijevic MR. Epidural and transcutaneous spinal electrical stimulation for restoration of movement after incomplete and complete spinal cord injury. Curr Opin Neurol. 2016;29:721-26.

10. Harkema S, Gerasimenko Y, Hodes J, Burdick J, Angeli C, Chen $\mathrm{Y}$, et al. Effect of epidural stimulation of the lumbosacral spinal cord on voluntary movement, standing, and assisted stepping after motor complete paraplegia: a case study. Lancet. 2011;377:1938-47.

11. Rattay F, Minassian K, Dimitrijevic M. Epidural electrical stimulation of posterior structures of the human lumbosacral cord: 2 . quantitative analysis by computer modeling. Spinal Cord. 2000;38:473-89.

12. Murray LM, Knikou M. Transspinal stimulation increases motoneuron output of multiple segments in human spinal cord injury. PLoS ONE. 2019;14. https://doi.org/10.1371/journal.pone. 0213696.

13. Murray LM, Tahayori B, Knikou M. Transspinal direct current stimulation produces persistent plasticity in human motor pathways. Sci Rep. 2018;8:717.

14. Angeli CA, Boakye M, Morton RA, Vogt J, Benton K, Chen Y, et al. Recovery of over-ground walking after chronic motor complete spinal cord injury. N Engl J Med. 2018;379:1244-50.

15. Wagner FB, Mignardot JB, Le Goff-Mignardot CG, Demesmaeker R, Komi S, Kappagrosso M, et al. Targeted neurotechnology restores walking in humans with spinal cord injury. Nature. 2018;563:65-71.
16. Minassian K, Jilge B, Rattay F, Pinter MM, Binder H, Gerstenbrand F, et al. Stepping-like movements in humans with complete spinal cord injury induced by epidural stimulation of the lumbar cord: electromyographic study of compound muscel action potentials. Spinal Cord. 2004;42:401-16.

17. Carhart MR, He J, Herman R, D’Luzansky S, Willis WT. Epidural spinal-cord stimulation facilitates recovery of functional walking following incomplete spinal-cord injury. IEEE Trans Neural Syst Rehabil Eng. 2004;12:32-42.

18. Hofstoetter US, Krenn M, Danner SM, Hofer C, Kern H, McKay $\mathrm{WB}$, et al. Augmentation of voluntary locomotor activity by transcutaneous spinal cord stimulation in motor-incomplete spinal cord-injured individuals: augmentation of locomotion by tSCS in incomplete SCI. Artif Organs. 2015;39:E176-86.

19. Gerasimenko YP, Lu DC, Modaber M, Zdunowski S, Gad P, Sayenko DG, et al. Noninvasive reactivation of motor descending control after paralysis. J Neurotrauma. 2015;32:1968-80.

20. Sayenko DG, Rath M, Ferguson AR, Burdiick JW, Havton LA, Edgerton VR, et al. Self-assisted standing enabled by non-invasive spinal stimulation after spinal cord injury. $\mathrm{J}$ Neurotrauma. 2018;36:1435-50.

21. Gorodnichev RM, Pivovarova EA, Puhov A, Moiseev SA, Savochin AA, Moshonkina TR, et al. Transcutaneous electrical stimulation of the spinal cord: a noninvasive tool for the activation of stepping pattern generators in humans. Hum Physiol. 2012;38:158-67.

22. Hofstoetter US, Freundl B, Binder H, Minassian K. Common neural structures activated by epidural and transcutaneous lumbar spinal cord stimulation: elicitation of posterior rootmuscle reflexes. PLoS ONE. 2018;13:e192013.

23. Behrman AL, Harkema SJ. Locomotor training after human spinal cord injury: a series of case studies. Phys Ther. 2000;80:688-700.

24. Minassian K, Hofstoetter US, Danner SM, Mayr W, Bruce JA, McKay WB, et al. Spinal rhythm generation by step-induced feedback and transcutaneous posterior root stimulation in complete spinal cord-injured individuals. Neurorehabil Neural Repair. 2016;30:233-43.

25. Bryce TN, Budh CN, Cardenas DD, Dijkers M, Felix ER, Finnerup NB, et al. Pain after spinal cord injury: an evidence-based review for clinical practice and research. Report of the National Institute on Disability and Rehabilitation Research Spinal Cord Injury Measures meeting. J Spinal Cord Med. 2007;30:421-40.

26. The SCIRE Research Team, Lam T, Noonan VK, Eng JJ. A systematic review of functional ambulation outcome measures in spinal cord injury. Spinal Cord. 2008;46:246-54.

27. Morganti B, Scivoletto G, Ditunno P, Ditunno JF, Molinari M. Walking index for spinal cord injury (WISCI): criterion validation. Spinal Cord. 2005;43:27-33.

28. van Hedel HJ, Wirz M, Dietz V. Assessing walking ability in subjects with spinal cord injury: validity and reliability of 3 walking tests. Arch Phys Med Rehabil. 2005;86:190-96.

29. van Hedel HJA, Wirz M, Curt A. Improving walking assessment in subjects with an incomplete spinal cord injury: responsiveness. Spinal Cord. 2006;44:352-56.

30. Dittuno P, Dittuno J. Walking index for spinal cord injury (WISCI II): scale revision. Spinal Cord. 2001;39:654-56.

31. Jackson A, Carnel C, Ditunno J, Read MS, Boninger ML, Schmeler MR, et al. Outcome measures for gait and ambulation in the spinal cord injury population. J Spinal Cord Med. 2008;31:487-99.

32. Biering-Sørensen F, Alai S, Anderson K, Charlifue S, Chen Y, DeVivo M, et al. Common data elements for spinal cord injury clinical research: a National Institute for Neurological Disorders and Stroke project. Spinal Cord. 2015;53:265-77.

33. Musselman KE. Clinical significance testing in rehabilitation research: what, why, and how? Phys Ther Rev. 2007;12:287-96. 
34. Musselman KE, Fouad K, Misiaszek JE, Yang JF. Training of walking skills overground and on the treadmill: case series on individuals with incomplete spinal cord injury. Phys Ther. 2009;89:601-11.

35. Lam T, Eng J, Wolfe D, Hsieh J, Whittaker M. A systematic review of the efficacy of gait rehabilitation strategies for spinal cord injury. Top Spinal Cord Inj Rehabil. 2007;13:32-57.
36. Burns AS, Delparte JJ, Patrick M, Marino RJ, Ditunno JF. The reproducibility and convergent validity of the walking index for spinal cord injury (WISCI) in chronic spinal cord injury. Neurorehabil Neural Repair. 2001;25:149-57.

37. Rejc E, Angeli CA, Atkinson D, Harkema SJ. Motor recovery after activity-based training with spinal cord epidural stimulation in a chronic motor complete paraplegic. Sci Rep. 2017;7:13476. 